\title{
Endobronchial Ultrasound-Guided Transbronchial Needle Aspiration of an Intravascular Sarcoma Metastasis
}

\author{
Frank Dusemund $^{\mathrm{a}}$ Tino Schneider ${ }^{\mathrm{a}}$ Christoph Zeise ${ }^{\mathrm{c}} \quad$ Christian Rothermundt $^{\mathrm{b}}$ \\ Thomas Kluckert $^{d}$ Sabine Schmid ${ }^{b}$ Martin H. Brutsche ${ }^{a}$ \\ Divisions of a Pneumology and ${ }^{b}$ Oncology and Hematology, Department of Internal Medicine, \\ cDepartment of Surgery and dinstitute for Radiology, Kantonsspital St. Gallen, St. Gallen, Switzerland
}

\section{Established Facts}

- The role of endobronchial ultrasound-guided transbronchial needle aspiration (EBUS-TBNA) in diagnosing endovascular lesions has rarely been described.

\section{Novel Insights}

- EBUS-TBNA seems to be a rapid, minimally invasive, safe and effective diagnostic procedure in selected cases of solid endovascular lesions.

\section{Key Words}

Endobronchial ultrasound-guided transbronchial needle aspiration · Pulmonary embolism · Endovascular lesions metastatic disease. EBUS-TBNA seems to be a rapid, minimally invasive, safe and effective diagnostic procedure in selected cases of endovascular lesions.

Copyright $\odot 2013$ S. Karger AG, Basel

\section{Abstract}

The role of endobronchial ultrasound-guided transbronchial needle aspiration (EBUS-TBNA) in the diagnosis of endovascular lesions has rarely been described. We report a case of EBUS-TBNA of a solid mass in the left pulmonary artery in a patient with synovial sarcoma of the kidney, which was performed without complications and led to the diagnosis of

\section{Introduction}

Endobronchial ultrasound-guided transbronchial needle aspiration (EBUS-TBNA) is a minimally invasive procedure utilized for the diagnosis of hilomediastinal lymph nodes and intrapulmonary lesions. Its role in di-

\section{KARGER}

E-Mail karger@karger.com www.karger.com/res
(C) 2013 S. Karger AG, Basel

0025-7931/13/0865-0430\$38.00/0 


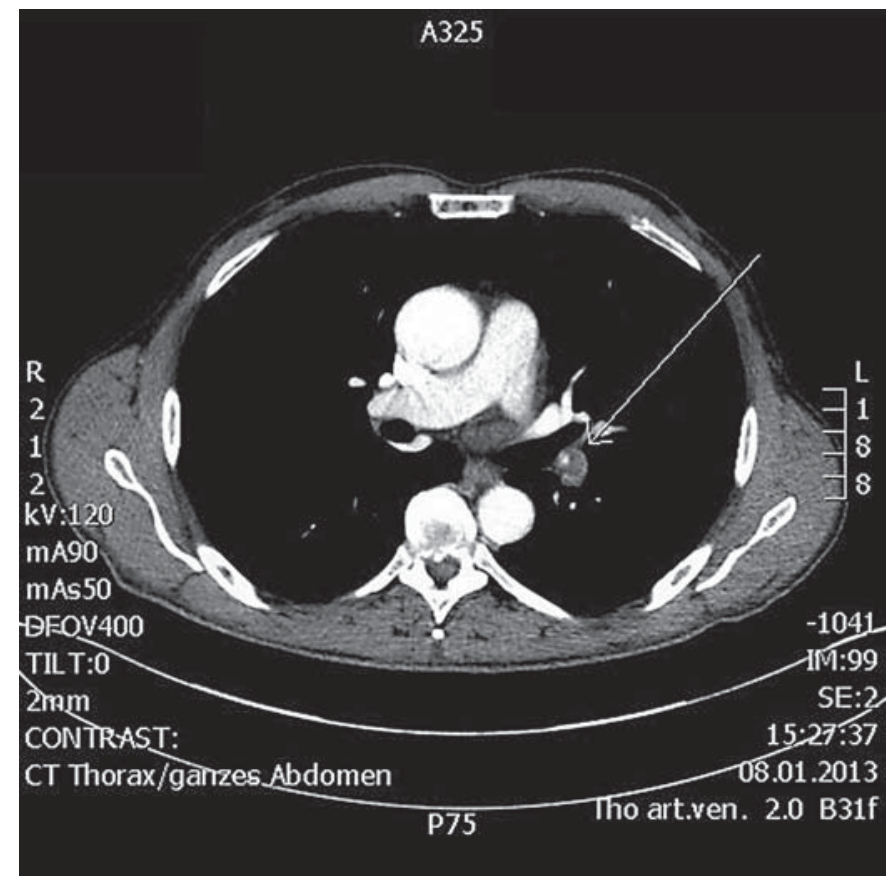

Fig. 1. Contrast-enhanced CT of the chest showing a perfusion defect in the left lower pulmonary artery (grey) with minimal remaining perfusion (white), indicated by the white arrow.

agnosing endovascular lesions has rarely been described [1-4]. We report a case of EBUS-TBNA of a solid mass in the left pulmonary artery in a patient with synovial sarcoma of the kidney.

\section{Case Report}

A 51-year-old patient with known synovial sarcoma of the right kidney, which had been treated with resection of the right kidney and ureter, followed by adjuvant chemotherapy 1 year before, was referred from oncology to our department for the evaluation of a new, occluding mass in the left pulmonary artery. This had been recognized by computed tomography (CT) of the chest (fig. 1, 2) and showed no increased metabolic activity in the positron emission tomography performed afterwards.

To perform flexible bronchoscopy, the patient was sedated with propofol via perfusor. In addition, $5 \mathrm{mg}$ of dihydrocodon were given to suppress coughing. Local anesthesia of the larynx, trachea and bronchi was conducted with lidocaine. Inspection of the bronchial tree was performed with the Olympus ${ }^{\circledR}$ BF H190 bronchoscope and showed no endobronchial abnormalities. For EBUS, we used the Olympus ${ }^{\circledR}$ BF UC 160F-OL8 bronchoscope. This showed a solid mass with perfusion defects in the left lower pulmonary artery, detected by Doppler ultrasound (fig. 3). EBUS-TBNA of this intraarterial solid mass was performed 3 -fold. The whole procedure lasted approximately $30 \mathrm{~min}$. Neither postinterventional bleeding nor

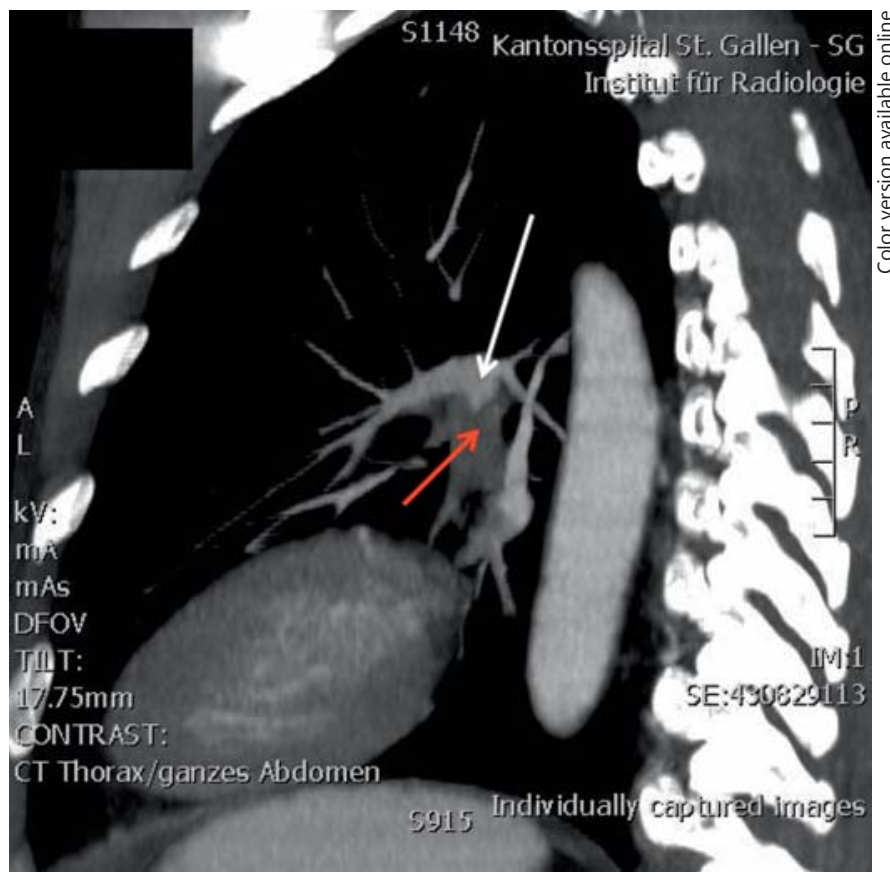

Fig. 2. Sagittal axis of contrast-enhanced CT of the chest showing the perfusion defect in the left lower pulmonary artery with distribution into subsegmental arteries, proving the intravascular localization (red arrow) and end of contrast agent (white arrow).

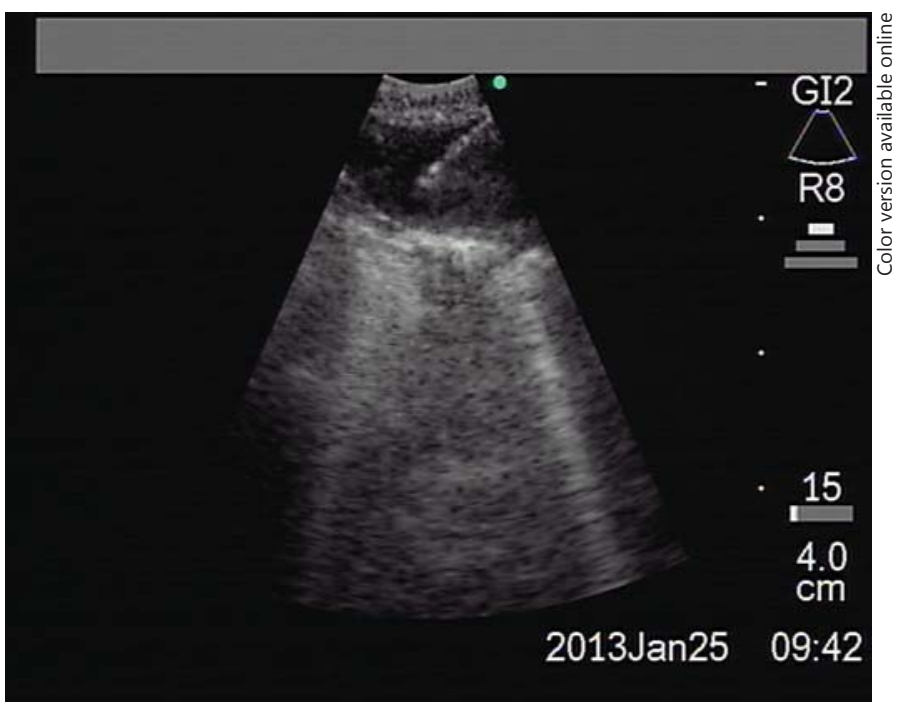

Fig. 3. EBUS-TBNA needle in the center of the lesion.

any other complications occurred. The contrast-enhanced chest CT, which had been performed before bronchoscopy, had not shown enlarged or suspect mediastinal lymph nodes or any other intravascular lesions than the one described. Therefore, we did not screen the other lymph node stations with EBUS. Due to the endo- 


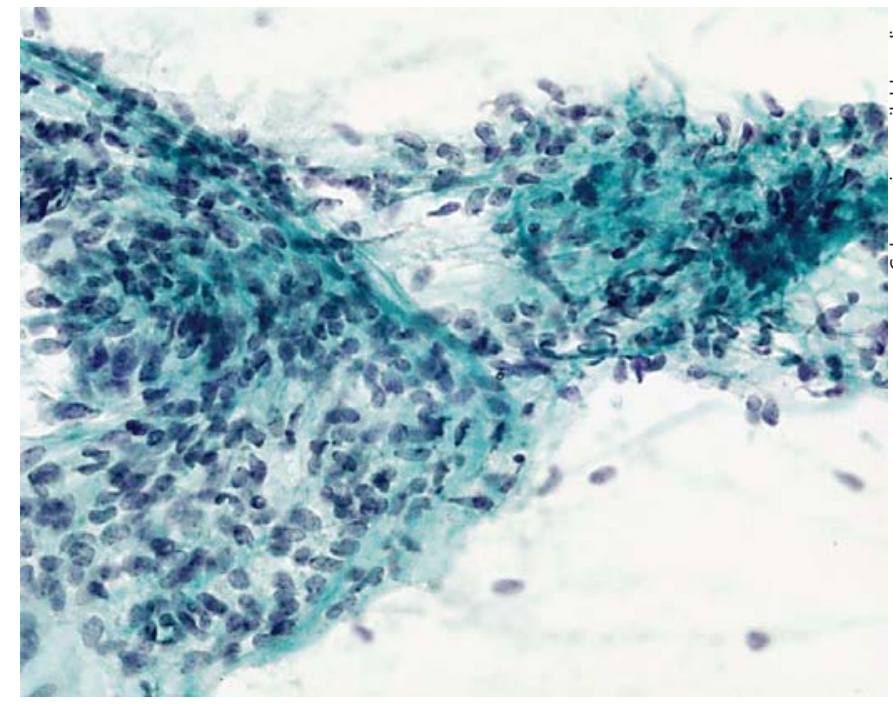

Fig. 4. Cytological smear of sarcoma metastasis.

vascular manipulation, a single intravenous antibiotic dose of $2.2 \mathrm{~g}$ amoxicillin with clavulanic acid was given. Cytopathological and molecular biological evaluation of the specimen could diagnose this mass as a metastasis of the known synovial sarcoma (fig. 4).

After an interdisciplinary discussion at the institutional tumor board for thoracic malignancies, resection of the left lower lobe and secondary resection of the lingula were performed. Histological evaluation confirmed the diagnosis of a singular metastasis of synovial sarcoma in the left pulmonary artery.

After complication-free R0 resection, the patient was referred for rehabilitation.

\section{Discussion}

In this case of an intravascular mass in the left lower pulmonary artery, which was suspicious of metastasis of synovial sarcoma of the kidney, EBUS-TBNA of this lesion could be performed without bleeding or infec- tious complications, and rapidly led to the correct diagnosis.

Only a few cases of EBUS-TBNA of endovascular lesions have been reported. Horowitz et al. [1] obtained diagnostic tissue from a mass in the left main pulmonary artery by using EBUS-TBNA without bleeding complications. Dhamija et al. [2] described a case of a 75-year-old woman with known infiltrating duct adenocarcinoma of the left breast, which had been diagnosed 15 years before, and then presented with hemoptysis. EBUS revealed the left hilar lymph node eroding into the left pulmonary artery, leading to a filling defect. This defect was sampled by EBUS-TBNA and diagnosed as metastasis of the breast carcinoma without complications. Chamorro et al. [3] reported a case of a 51-year-old woman with pulmonic tumor embolism 5 years after diagnosis and treatment of retroperitoneal synovial sarcoma. This also could be sampled by EBUS-TBNA and led to the diagnosis of metastatic disease without any complications. Park et al. [4] reported 2 cases of pulmonary artery sarcoma, which had safely been sampled and diagnosed by EBUS-TBNA.

Another implication of EBUS could be the assessment of pulmonary circulation, e.g. in the diagnosis of pulmonary embolism (PE). In 2009, Aumiller et al. [5] performed a pilot study regarding this issue with 32 patients, in whom $96 \%$ of CT-documented PE could be detected by EBUS without complications within a short procedure time between 3 and 5 min. Egea Santaolalla et al. [6] reported a case in 2011, where they could diagnose PE with EBUS, whereas the thrombus had not previously been detected by CT scan. In a recent study, Sentürk et al. [7] diagnosed PE with EBUS in $8 / 8$ cases, which had been documented by CT scan.

In conclusion, EBUS-TBNA seems to be a rapid, minimally invasive, safe and effective diagnostic procedure in selected cases of solid endovascular lesions. In addition, it could be useful in cases of suspected PE with contraindications for contrast-enhanced CT.

\section{References}

1 Horowitz JC, Kleaveland K, Arenberg D: Endobronchial biopsy of an intrapulmonary arterial mass. J Bronchology Interv Pulmonol 2013;20:93-95.

-2 Dhamija A, Agarwal A, Basu A, et al: Hilar lymph node eroding into the pulmonary artery diagnosed by endobronchial ultrasoundguided transbronchial needle aspiration. BMJ Case Rep 2012; doi:10.1136/bcr-2012-007438.
3 Chamorro N, Blanco I, Sánchez M, et al: The expanding horizons of endobronchial ultrasound: diagnosis of a tumor embolism. Chest 2012;142:1334-1336.

4 Park JS, Chung JH, Jheon S, et al: EBUSTBNA in the differential diagnosis of pulmonary artery sarcoma and thromboembolism. Eur Respir J 2011;38:1480-1482.

5 Aumiller J, Herth FJ, Krasnik M, et al: Endobronchial ultrasound for detecting central pulmonary emboli: a pilot study. Respiration 2009;77:298-302.
Dusemund/Schneider/Zeisel/ Rothermundt/Kluckert/Schmid/Brutsche 